\title{
Study of antibiotic prescribing among dental practitioners in Shiraz, Islamic Republic of Iran
}

\author{
G. Vessal, ${ }^{1}$ A. Khabiri, ${ }^{2}$ H. Mirkhani, ${ }^{3}$ B.D. Cookson ${ }^{4}$ and M. Askarian ${ }^{2}$
}

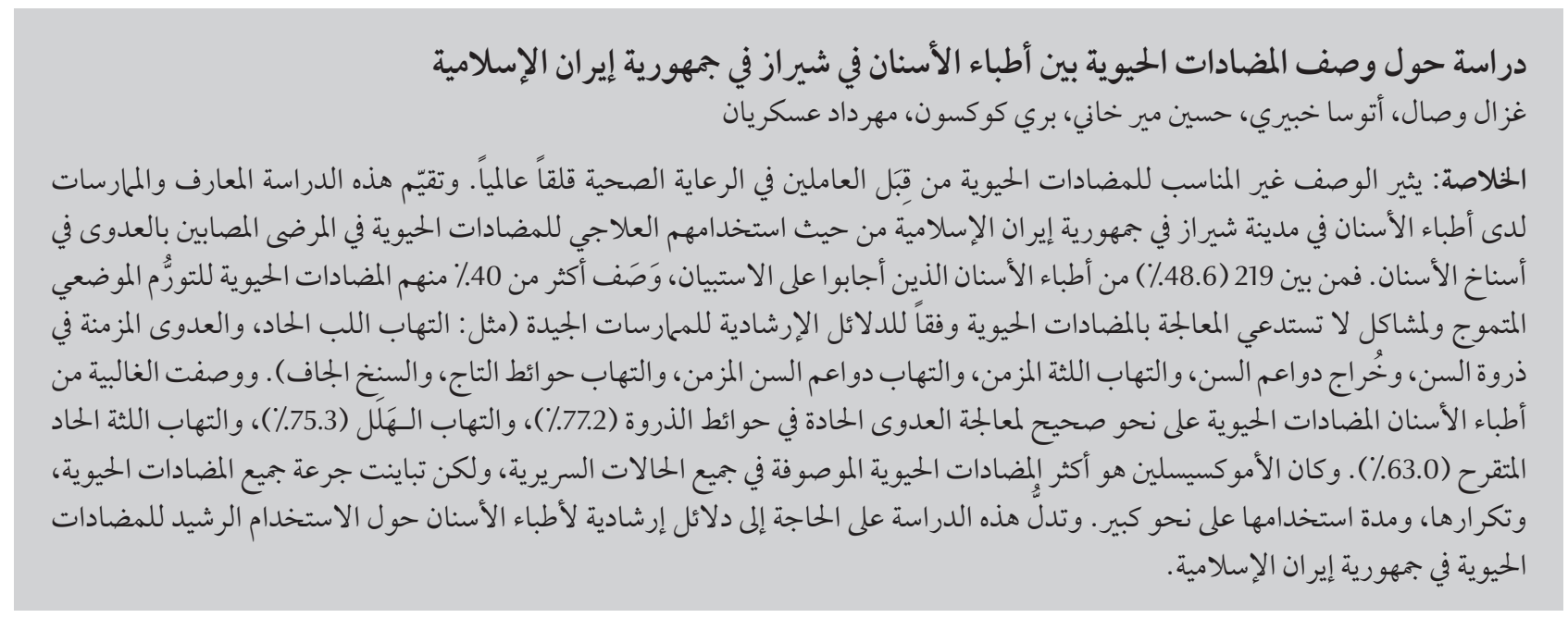

ABSTRACT Inappropriate prescribing of antibiotics by health care professionals is a worldwide concern. This study evaluated the knowledge and practices of dental practitioners in the city of Shiraz, Islamic Republic of Iran regarding their therapeutic use of antibiotics for patients with dentoalveolar infections. Of 219 (48.6\%) dentists responding to the questionnaire more than $40 \%$ would prescribe antibiotics for localized fluctuant swelling and for problems for which antibiotics are not required according to good practice guidelines (acute pulpitis, chronic apical infection, periodontal abscess, chronic gingivitis, chronic periodontitis, pericoronitis and dry socket). A majority correctly prescribed antibiotics for acute periapical infection (77.2\%), cellulitis (75.3\%) and acute ulcerated gingivitis (63.0\%). Amoxicillin was the most frequently prescribed antibiotic for all clinical conditions but there was a wide variation in dosage, frequency and duration for all antibiotics used. Guidelines on rational antibiotic use are needed for dental practitioners in the Islamic Republic of Iran.

\section{Étude de la prescription d'antibiotiques par des dentistes à Chiraz (République islamique d'Iran)}

RÉSUMÉ La prescription inadaptée d'antibiotiques par les professionnels de la santé est un sujet de préoccupation mondial. La présente étude a évalué les connaissances et les pratiques de dentistes dans la ville de Chiraz (République islamique d'Iran) concernant leur utilisation thérapeutique des antibiotiques pour les patients atteints d'infections dento-alvéolaires. Sur 219 dentistes $(48,6 \%)$ ayant répondu au questionnaire, plus de $40 \%$ prescriraient des antibiotiques pour un odème fluctuant localisé et d'autres problèmes pour lesquels les antibiotiques ne sont pas nécessaires selon les directives sur les bonnes pratiques (pulpite aiguë, pathologie apicale chronique, abcès périodontal, gingivite chronique, périodontite chronique, péricoronarite et alvéolite sèche). La majorité d'entre eux prescrivent à bon escient des antibiotiques pour une infection périapicale aiguë (77,2 \%), un phlegmon (75,3\%) et une gingivite ulcéreuse aiguë (63,0 \%). L'amoxicilline est l'antibiotique le plus fréquemment prescrit pour toutes les affections cliniques. En revanche, la posologie, la fréquence et la durée de l'ensemble des traitements antibiotiques varient considérablement. Des directives sur l'utilisation rationnelle des antibiotiques sont nécessaires pour les dentistes en République islamique d'Iran.

'Department of Clinical Pharmacy, Faculty of Pharmacy; ${ }^{2}$ Department of Community Medicine; ${ }^{3}$ Department of Pharmacology, Shiraz University of Medical Sciences, Shiraz, Islamic Republic of Iran (Correspondence to M. Askarian: mehrdadaskarian@gmail.com).

${ }^{4}$ Laboratory of Health Care Associated Infection, Health Protection Agency, London, United Kingdom.

Received: 06/11/09; accepted: 18/03/10 


\section{Introduction}

Dental practitioners regularly prescribe antibiotics for therapeutic or prophylactic purposes to manage oral and dental infections. However, inappropriate prescribing and excessive use of antibiotics have been identified as major factors in the emergence of antibiotic resistance, which is an ongoing challenge ever since the discovery of antimicrobial agents [1]. There are other issues too, such as possible adverse events and additional costs of prescribing. Consequently, surveillance of antimicrobial resistance, monitoring of antibiotic usage and attempts to improve prescribing attitudes have become crucial [2].

In 1997 more than 3.5 million prescriptions for antibiotics were dispensed by general dental practitioners in England at a net ingredient cost of $£ 5.2$ million. Antibiotic prescribing by dentists may therefore play a major role in the emergence of resistant bacterial strains, particularly when there is evidence of misuse [3].

Currently there are no specific guidelines in Islamic Republic of Iran for prophylactic or therapeutic prescribing of antibiotics in dentistry. In addition, there are no data describing the indications for which antibiotics are prescribed, whether these have any scientific basis, or whether the antibiotic agents, their dose, frequency and duration, are based on published guidelines or standards. We therefore conducted this study to evaluate the knowledge and attitude of dental practitioners in the city of Shiraz (the capital of Fars province) regarding their therapeutic use of antibiotics for patients with dentoalveolar infections.

\section{Methods}

\section{Sample}

We estimated the sample size of 197 using $d=0.3$, standard deviation $=$ $1.5, \alpha=0.05$ and $\beta=0.2$. We mailed questionnaires to all dentists in the study area $(n=450)$, which according to our previous studies would enable us to reach the calculated sample size.

\section{Questionnaire}

The questionnaire was a modification of that described by Palmer et al. [3] and aimed to investigate the knowledge of dental practitioners about therapeutic prescribing of antibiotics. To ensure its validity the questionnaire was piloted and discussed with 12 dental specialists from each department of the School of Dentistry in Shiraz. In order to evaluate the reliability of the questionnaire, an extended pilot study was performed on 50 dental practitioners. The KuderRichardson coefficient of reliability was high $(r=0.86)$. Thus no changes were made to the questionnaire.

The questionnaires were mailed out to the selected dental practitioners in the city of Shiraz over a 3-week period in 2006, and collected 1 week later from their offices. It recorded the age, sex, practitioners' final degree (general or specialist), when and where it was obtained, whether the practitioner was affiliated to the university and any participation in the last 2 years in continuing education programmes related to the rational use of antibiotics. Respondents answered anonymously and were requested to avoid use of any reference materials while answering the questionnaire.

The questionnaire also explored the number of patients treated per week and for which clinical signs the practitioner would prescribe antibiotics for patients presenting with a dental infection. The clinical signs chosen were: elevated temperature and evidence of systemic spread; localized fluctuant swelling; gross or diffuse swelling; restricted mouth opening; difficulty in swallowing; and closure of the eye due to swelling. We also explored whether patients' expectations of an antibiotic prescription would be a reason for prescribing antibiotics.
Another part of the questionnaire investigated the use of antibiotics for common clinical conditions. If a positive response was given, the practitioners were asked to state the antibiotic they would prescribe, its dose, interval and duration, for patients who were not allergic to penicillin. The practitioner was also asked what antibiotic s/he would choose if the patient was allergic to penicillin. The clinical conditions were acute pulpitis, acute periapical infection, chronic apical infection, periodontal abscess, acute ulcerative gingivitis, chronic gingivitis, chronic periodontitis, pericoronitis, dry socket, and cellulitis.

In reviewing the literature, a systematic search for literature published in English available via MEDLINE for the years 1996 through December 2007 was conducted. The search terms included: "antibiotic treatment and dentistry". Identified articles were used to select additional key terms for further searches. Other relevant articles were identified from the bibliographies of these papers. A search was also conducted of the US Centers for Disease Control and Prevention and World Health Organization published documents, which focused on antibiotic treatment and dentistry. We also searched several leading textbooks on this subject.

\section{Data analysis}

A knowledge score was constructed for each question asked regarding signs in which the practitioner would prescribe antibiotics. Each of these 6 questions were graded as 0 (if incorrect) versus 1 (if correct). For questions regarding use of antibiotics in common clinical conditions, knowledge of respondents was evaluated based on evidence-based guidelines and standards in selected published literature.

Means and standard deviations (SD) were obtained for continuous variables, and compared using Student $t$-test. Categorical variables were reported as percentages and compared using the chi-squared test. Non-parametric 
correlation was used to determine the strength of the relationship between demographic and professional factors and the knowledge score. The level of statistical significance of all tests was a 2 -tailed $P$-value $<0.05$. All analyses were performed using SPSS, version 9.

\section{Results}

\section{Background characteristics}

Of the 450 dental practitioners to whom the questionnaires were sent, 219 (48.6\%) completed the forms (64.4\% male, $35.6 \%$ female). The majority of the practitioners graduated from Shiraz Dental School (66.2\%). Only $5.9 \%$ of the practitioners had attended a continuing education programme on rational use of antibiotics within the previous 2 years. The demographic and professional characteristics of respondents are shown in Table 1.

\section{Prescribing habits}

Table 2 shows the clinical signs for which the practitioners would prescribe antibiotics. Over $80 \%$ would prescribe for patients with elevated body temperature, gross or diffuse facial swelling and closure of the eye due to swelling. A minority of practitioners (17.7\%) would prescribe antibiotics as a result of a patient's demand.

Regarding antibiotic prescriptions for clinical signs, the mean knowledge score was 3.9 (range 0-6). There was no statistical significant difference in the mean knowledge scores by sex, professional qualifications or having attended a continuing education course (Table 3).

\section{Prescribing for specific conditions}

The percentage of practitioners prescribing for specific conditions are shown in Table 4. The results showed that the majority of dentists would prescribe for acute periapical infection (77.2\%), cellulitis (75.3\%) and acute

\begin{tabular}{lcc}
\hline $\begin{array}{l}\text { Table 1 Demographic and professional characteristics of participating dental } \\
\text { practitioners }(\boldsymbol{n}=\mathbf{2 1 9})\end{array}$ & Value \\
\hline Variable & No. & $\%$ \\
\multicolumn{1}{l}{ Sex } & 141 & 64.4 \\
$\quad$ Male & 78 & 35.6 \\
$\quad$ Female & & \\
Degree & 186 & 84.9 \\
$\quad$ General practitioner & 33 & 15.1 \\
$\quad$ Specialist & 14 & 6.5 \\
Academic position & 205 & 93.5 \\
$\quad$ Yes & Mean (SD) & Range \\
No & $36.5(7.8)$ & $24-72$ \\
& $9.6(7.0)$ & $0.5-37$ \\
Age (years) & $35.7(27.5)$ & $5-200$ \\
Duration of practice (years) & \\
No. of patients/week &
\end{tabular}

$S D=$ standard deviation. ulcerated gingivitis (63.0\%). The practitioner's preferred choice of antibiotics for specific conditions that actually need antibiotic treatment, assuming no allergy to penicillin, is shown in Table 5. Amoxicillin was the antibiotic most frequently prescribed.

The dose, frequency and duration of treatment with each antibiotic prescribed differed among the practitioners. Of the 126 practitioners who would prescribe amoxicillin for acute periapical infection, the great majority (70.6\%) chose a 250 or $500 \mathrm{mg}$ dose $3 \times$ daily for a period of $6-10$ days. Few dentists (6.3\%) chose the lower dose of 250 $\mathrm{mg}$ and only $2.4 \%$ preferred a shorter duration of 2-3 days. Some practitioners (10.3\%) even chose a duration of 4 weeks. Of those 37 practitioners who prescribed penicillin $\mathrm{V}$ for acute periapical infection, more than $81.1 \%$ chose a 250 or $500 \mathrm{mg}$ dose $4 \times$ daily for $6-10$ days but only $8.1 \%$ chose the lower dose.

For acute ulcerative gingivitis, more than half of the 61 practitioners who prescribed amoxicillin (52.5\%) chose a 500 mg dose $3 \times$ daily for a period of 6-10 days; only $3.3 \%$ chose a shorter duration of 2-3 days and $16.4 \%$ even chose a duration of 4 weeks. Of 38 practitioners who chose metronidazole to treat acute ulcerative gingivitis, $50.0 \%$ chose a 250

\begin{tabular}{|c|c|c|}
\hline \multirow[t]{2}{*}{ Clinical sign } & \multicolumn{2}{|c|}{ Would prescribe } \\
\hline & No. & $\%$ \\
\hline Gross diffuse swelling ${ }^{\mathrm{a}}$ & 193 & 88.3 \\
\hline Elevated temperature and evidence of systemic spread ${ }^{a}$ & 185 & 84.4 \\
\hline Closure of the eye because of swelling ${ }^{a}$ & 178 & 81.4 \\
\hline Difficulty in swallowing & 103 & 46.9 \\
\hline Localized fluctuant swelling & 101 & 46.0 \\
\hline Restricted mouth opening & 94 & 42.7 \\
\hline Patient's expectation for a prescription & 39 & 17.7 \\
\hline
\end{tabular}

${ }^{a}$ Clinical signs that that need antibiotic treatment. 


\begin{tabular}{lrcc}
\hline $\begin{array}{l}\text { Table } 3 \text { Knowledge score by professional and demographic variables for clinical } \\
\text { signs that need antibiotics }\end{array}$ & No. & $\begin{array}{c}\text { Mean (SD) } \\
\text { score }^{b}\end{array}$ & P-value \\
\hline Variable & 139 & $3.9(1.2)$ & 0.76 \\
Sex & 77 & $3.9(1.2)$ & \\
$\quad$ Male & & & 0.53 \\
Female & 180 & $3.9(1.2)$ & 0.98 \\
Degree & 32 & $3.8(1.4)$ & \\
General & 14 & $3.9(1.6)$ & 0.18 \\
Specialist & 203 & $3.9(1.2)$ & \\
Academic & & & \\
Non-academic & 13 & $4.4(1.1)$ & \\
Continuing education course ${ }^{a}$ & 197 & $3.9(1.2)$ & \\
Yes & 219 & $3.9(1.3)$ & \\
No &
\end{tabular}

${ }^{a}$ Attended continuing education programme on rational use of antibiotics within the previous 2 years. ${ }^{b}$ Range: $0-6$.

$S D=$ standard deviation

mg dose $3 \times$ daily for $6-10$ days, $34.2 \%$ incorrectly chose $4 \times$ daily interval for this drug and only about $7.9 \%$ would treat for 2-3 days. Practitioners who chose tetracycline for the treatment of this condition used a variety of different dosages, durations and intervals.

For the treatment of cellulitis, a majority of the 85 practitioners who prescribed amoxicillin $(64.7 \%)$ chose a $500 \mathrm{mg}$ dose $3 \times$ daily for $6-10$ days and $21.2 \%$ chose an even higher duration of treatment (4 weeks). Most of the 21 practitioners who chose penicillin $\mathrm{V}$ to treat this condition chose a $500 \mathrm{mg}$ dose $4 \times$ daily for $7-10$ days (57.1\%), while $19.0 \%$ preferred 4 weeks of treatment. A considerable proportion of the practitioners surveyed preferred an injection of penicillin to treat this condition; however, there was considerable disagreement regarding the dosage and the number of injections.

For patients allergic to penicillin, erythromycin was the most common antibiotic prescribed by the respondents $(70.0 \%)$, followed by clindamycin (15.7\%) and cephalexin (7.6\%). Of

\begin{tabular}{lcc}
\hline $\begin{array}{l}\text { Table } 4 \text { Prescribing of antibiotics by dental practitioners for selected dental } \\
\text { diagnoses }(\boldsymbol{n}=\mathbf{2 1 9})\end{array}$ & \multicolumn{2}{c}{ Would prescribe $^{\text {No. }}$} \\
\hline Diagnosis & 169 & 77.2 \\
\hline Acute periapical infection $^{\mathrm{a}}$ & 165 & 75.3 \\
Cellulitis $^{\mathrm{a}}$ & 147 & 67.1 \\
Pericoronitis $_{\text {Acute ulcerative gingivitis }}^{\mathrm{a}}$ & 138 & 63.0 \\
Periodontal abscess $_{\text {Chronic apical infection }}$ & 134 & 61.2 \\
Acute pulpitis & 72 & 32.9 \\
Chronic periodontitis & 55 & 25.1 \\
Dry socket & 45 & 20.5 \\
Chronic gingivitis & 45 & 20.5 \\
\hline
\end{tabular}

${ }^{a}$ Diagnoses that need antibiotic treatment. those practitioners who preferred erythromycin more than half chose a 400 $\mathrm{mg}$ dose $4 \times$ daily for $6-10$ days. Others incorrectly prescribed a $3 \times$ daily dose, or a longer duration of treatment of up to 4 weeks.

\section{Discussion}

Within the last few decades antimicrobial resistance has become a worldwide problem and constitutes a major threat to public health. The unsystematic prescribing of antibiotics by health care professionals is a major factor to be considered. Evidence of the inappropriate use of antibiotics in dentistry has increased and this could lead to the problem of antimicrobial resistance [3-6]. This fact and the increase in the number of antibiotic prescriptions written by dentists each year [4] shows the importance of examining the role of dentists in prescribing antibiotics in everyday practice.

The present study showed evidence of poor prescribing of antibiotics by dentists in Shiraz. The indications for antibiotics in acute dentoalveolar infections have been defined as: signs of spreading infection, patient malaise, temperature elevation and lymphadenitis $[3,4]$. Generally the survey showed that dental practitioners are aware of these indications and mostly used antibiotics wisely for acute infections. However, more than $40 \%$ would prescribe antibiotics for localized fluctuant swelling. Therefore, a considerable proportion of practitioners prescribed antibiotics for all swellings where local treatment would have been sufficient. This was a similar finding to studies performed in Kuwait [6], and Yemen [5], where $55 \%$ and $68 \%$ of the practitioners respectively prescribed antibiotics for local swelling. With a mean score of 3.9 out of a maximum possible score of 6 , we could say that our dental practitioners had an intermediate knowledge regarding clinical signs that require antibiotic treatment, a score 


\begin{tabular}{|c|c|c|}
\hline \multicolumn{3}{|c|}{$\begin{array}{l}\text { Table } 5 \text { Antibiotics preferred by dental practitioners for clinical conditions that } \\
\text { need antibiotic therapy }\end{array}$} \\
\hline \multirow[t]{2}{*}{ Clinical condition } & \multicolumn{2}{|c|}{ Would prescribe } \\
\hline & No. & $\%$ \\
\hline \multicolumn{3}{|c|}{ Acute periapical infection $(n=169)^{a}$} \\
\hline Amoxicillin & 126 & 74.5 \\
\hline Penicillin V & 37 & 21.9 \\
\hline Other ${ }^{\mathrm{b}}$ & 6 & 3.5 \\
\hline \multicolumn{3}{|c|}{ Acute ulcerative gingivitis ( $n=138$ ) } \\
\hline Amoxicillin & 61 & 44.2 \\
\hline Metronidazole & 38 & 27.5 \\
\hline Tetracycline & 18 & 13.0 \\
\hline Other ${ }^{c}$ & 21 & 15.2 \\
\hline \multicolumn{3}{|l|}{ Cellulitis $(n=165)$} \\
\hline Amoxicillin & 85 & 51.5 \\
\hline Penicillin & 40 & 24.2 \\
\hline Penicillin V & 21 & 12.7 \\
\hline Other ${ }^{d}$ & 19 & 11.5 \\
\hline
\end{tabular}

${ }^{a}$ Number of dentists who would prescribe an antibiotic for this condition.

${ }^{b}$ Penicillin V + metronidazole, metronidazole, cephalexin; ${ }^{c}$ Penicillin V, ampicillin, cephalexin, erythromycin, doxycycline; ${ }^{d}$ Cephalexin, ampicillin, metronidazole, coamoxiclav, gentamicin.

similar to the Yemeni general dentists (mean score of 3.7) [5]. It is gratifying to note that only a minority of the respondents (17.7\%) would prescribe antibiotics for an unscientific reason such as patients' expectations, which is irrational and an abuse of antibiotic usage. This figure was found to be only $4 \%$ for dental practitioners in Kuwait [6], and 10\% for the dentists in England [3], but was similar to dental practitioners practising in Yemen (15\%) [5].

The proportion of practitioners who would routinely prescribe antibiotics for specific conditions varied a great deal among the presenting diseases. More than $70 \%$ of those surveyed would correctly prescribe antibiotics for cellulitis and acute periapical infections, while only $63 \%$ would prescribe antibiotics for acute ulcerative gingivitis, which is recommended as part of the initial therapy [4]. The majority of uncomplicated infected swellings of dental origin can be successfully treated by removing the source of infection by drainage of abscesses, removal of infected pulp contents or tooth extraction. Antibiotics are not effective in the management of pain associated with irreversible pulpitis $[4,7]$. However, $25.0 \%$ of our dental practitioners surveyed believed in the use of antibiotics in patients presenting with acute pulpitis. This was similar to the study performed in Yemen and $\mathrm{Ku}$ wait in which $32 \%$ and $20 \%$ of dentists respectively would prescribe antibiotics for this condition $[5,6]$. However, a lower percentage (13\%) of dental practitioners in England prescribed antibiotics for this acute pulpitis.

Chronic apical infections rarely need antibiotics unless there is evidence of gross local spread; extraction or root canal therapy are the definitive treatment options. In this survey about one-third of the practitioners would prescribe antibiotics for chronic apical infections, similar to the studies in Kuwait and England [3,6]. However, our practitioners' knowledge in this regard was much higher than the Yemeni dental practitioners in whom $72 \%$ would prescribe for chronic apical infections. Chronic dentoalveolar infections rarely require antibiotics, unless there is evidence of gross local spread [4], and the majority of patients with the commonly encountered forms of chronic periodontitis usually respond well to conventional therapies [8]. Adjunctive antibiotics should be prescribed only for a very limited group of patients - e.g. those with specific clinical features or aggressive forms of periodontal disease - in order to prevent antibiotic resistance due to periodontal therapy [9]. Nevertheless about 14\% and $20 \%$ respectively of our dental practitioners surveyed would prescribe antibiotics routinely for chronic gingivitis and chronic periodontitis. These results were similar to those obtained from dental practitioners in Kuwait [6]. Our dental practitioners were more knowledgeable in this regard compared with the dental practitioners in Yemen, where $54 \%$ and $66 \%$ respectively would routinely prescribe antibiotics for these infections [5]. A lower percentage (3\% and $13 \%$ respectively) of dental practitioners in England would prescribe antibiotics in the above conditions [3]

Pericoronitis, periodontal abscesses and dry sockets are treated by local measures, and antibiotics are only indicated for large spreading infections or systemic involvement $[4,10]$. The use of systemic antibiotics in the treatment of periodontal disease is controversial. It is acceptable not to use antibiotics routinely for the treatment of adult periodontal disease. Mechanical debridement methods, including drainage of pus for acute periodontal abscesses, should be considered the first-line treatment for most periodontal diseases. Systemic antimicrobials should be considered as adjuncts to such methods, and never used alone in this chronic disease, as they can predispose to abscess formation. Adjunctive systemic antimicrobials may be considered in acute disease where debridement or drainage of pus is difficult, or there is local spread or systemic upset. There is also evidence that systemic antibiotics can be used in juvenile periodontitis $[4,11]$. Initial treatment of pericoronitis is usually aimed at debridement of the 
periodontal pocket by irrigation or by mechanical means, disinfection of the pocket with an irrigation solution such as hydrogen peroxide or chlorhexidine, and surgical management by extraction of the opposing maxillary third molar, and occasionally, of the offending mandibular third molar. Severe cases of pericoronitis with systemic symptoms may warrant antibiotic therapy [12]. Such exceptional situations were not covered in our questionnaire, and unfortunately more than $60 \%$ of our dental practitioners surveyed would prescribe antibiotics routinely for periodontal abscess and pericoronitis. In the study performed in England and Yemen more than 80\% of the practitioners, would prescribe for these two conditions [3], while this percentage was more than $70 \%$ in Kuwait [6]. The percentage of our practitioners prescribing antibiotics for dry socket was $20 \%$, much lower compared with the studies performed in Kuwait, Yemen and England $(>50 \%)[3,5,6]$.

Amoxicillin was the most frequently prescribed antibiotic for acute dentoalveolar infections requiring antibiotics, which is similar to other studies $[3,6,13]$. This was followed by penicillin $\mathrm{V}$ for acute periapical infections, metronidazole for acute ulcerative gingivitis and penicillin injection for cellulitis (Table $3)$. The data revealed that the dentists surveyed prescribed a wide spectrum of antibiotics. The use of penicillin $\mathrm{V}$ is based on old studies that have isolated mainly streptococci and staphylococci from dental abscesses, while more recent studies have shown that the main isolates from dental abscesses are complex mixtures of facultative and anaerobic bacteria, some of which are penicillin resistant $[14,15]$. Based on a review of the evidence and good practice for prescribing therapeutic antibiotics in dentistry [4], amoxicillin $250 \mathrm{mg}$ $3 \times$ daily for a maximum of 5 days and metronidazole $200 \mathrm{mg} 3 \times$ daily for 3 days have been recommended as first and second choice treatments respectively. For patients allergic to penicillin, erythromycin $250 \mathrm{mg} 4 \times$ daily or 500 mg $2 \times$ daily up to 4 days have been recommended [4].

In agreement with previous studies $[13,16,17]$, there was a considerable variation from the recommended frequencies, doses and duration of antibiotic therapy. There is increasing evidence that short courses of antibiotics together with local surgical measures are adequate for treating dentoalveolar infections $[4,18,19]$. Prolonged courses of antibiotics, which were recommended by most of the practitioners in our survey for periods up to 10 days, could be harmful, due to the fact that the dose and duration of therapy are key factors in developing antibiotic resistance. The fact that a considerable number of our practitioners surveyed chose a duration of treatment of up to 4 weeks is of great concern. Acute orofacial infections have a rapid onset and relatively short duration of 2 to 7 days, particularly if the offending cause is treated and/or eliminated [20]. If clinical experience and the nature of the infection demonstrate that its predicted course may be 3 days, then 3 days of antibiotic therapy is enough. When clinical evidence indicates that the infection is expected to resolve or is resolved, the antibiotic therapy should be terminated $[4,19,20]$.

Unfortunately the optimal duration of antibiotic therapy for many dental infections has never been defined by randomized controlled trials. Current guidelines are based on expert opinion, which is considered to be the lowest level of evidence. There is an urgent need for randomized controlled trials with the objective of providing a scientific basis for best practice recommendations. Until such data exist, the antibiotics should be applied for a short duration. It is believed that large doses of amoxicillin $(500 \mathrm{mg})$, which was prescribed by a majority of our practitioners, are not necessary in acute dentoalveolar infections, as the absorption of this antibiotic in standard $250 \mathrm{mg}$ amounts is good enough to be therapeutically effective
$[3,4]$. The dose of metronidazole prescribed by our dental practitioners was $250 \mathrm{mg}$ (in contrast with the recommended dose of $200 \mathrm{mg}$ [4]), due to the fact that in the Islamic Republic of Iran the tablets are formulated at this higher dosage level.

Our findings indicate that the scientific basis for prescribing antimicrobial agents was neglected by the majority of the respondents. Most of those surveyed used antibiotics routinely for conditions where local treatment would be sufficient. This is not surprising as similar findings were reported among other health professionals in Islamic Republic of Iran [21-23], and by dental practitioners in other countries $[3,5,6]$. It is clear that our dental practitioners need expert advice on when and what to prescribe, for how long and in what dosage. Qualitative research is required to see if we can find out directly from practitioners why this problem of inappropriate antibiotic prescribing is so intractable, as well as the practitioners' attitude towards changing their prescribing behaviour.

Audit of clinical antibiotic prescribing in dentistry has been reported to improve general dental practitioners' attitudes to prescribing antimicrobials, reducing the number of prescriptions following the introduction of guidelines $[24,25]$. It is important to inform the dental community about the accepted current antibiotic prescription guidelines and the related evidence-based clinical practice and this paper adds to the evidence needed for designing national guidelines for our dental practitioners. There is also a need to improve undergraduate education and to increase postgraduate courses and other educational activities on antibiotic prescribing, especially since there was no difference in prescribing between those practitioners who had completed a continuing education programme and those who had not. This study lends support to the hypothesis that antibiotics are being inappropriately 
prescribed by the dental profession in Islamic Republic of Iran. The response rate to the questionnaire was poor and, as those who responded were likely to be more knowledgeable about the issue, the actual level of knowledge among dentists in this area may be even lower. Introducing guidelines and re-auditing after a few years would be an important step in implementing rational antibiotic use.

\section{Acknowledgements}

This study was funded by the Deputy for Research at the Shiraz University of Medical Sciences

\section{References}

1. Weber JT, Courvalin P. An emptying quiver: antimicrobial drugs and resistance. Emerging Infectious Diseases, 2005, 11:791-793

2. Al-Haroni $\mathrm{M}$, Skaug $\mathrm{N}$. Incidence of antibiotic prescribing in dental practice in Norway and its contribution to national consumption. Journal of Antimicrobial Chemotherapy, 2007, 59:1161-1166.

3. Palmer NAO et al. A study of therapeutic antibiotic prescribing in National Health Service general dental practice in England. British Dental Journal, 2000, 188:554-558.

4. Palmer NAO. Revisiting the role of dentists in prescribing antibiotics. Dental Update, 2003, 30:570-574.

5. Al-Haroni M, Skaug N. Knowledge of prescribing antimicrobials among Yemeni general dentists. Acta Odontologica Scandinavica, 2006, 64:274-280.

6. Salako NO et al. Pattern of antibiotic prescription in the management of oral diseases among dentists in Kuwait. Journal of Dentistry, 2004, 32:503-509.

7. Keenan JV et al. Antibiotic use for irreversible pulpitis. Cochrane Database of Systematic Reviews, 2005, CD004969.

8. Newman MG, Takei HH, Fermic AC, eds. Carranza's clinical periodontology, 9th ed. Philadelphia: Saunders, 2002

9. Walter C. Weiger R. Antibiotics as the only therapy of untreated chronic periodontitis: a critical commentary. Journal of Clinical Periodontology, 2006. 33:938-939.

10. Faculty of Dental Surgery. National clinical guidelines. London, Royal College of Surgeons of England, 1997.

11. Addy M, Martin MV. Systemic antimicrobials in the treatment of chronic periodontal disease: a dilemma. Oral Diseases, 2003, 9(Suppl. 1):38-44.

12. Miloro M et al., eds. Peterson's principles of oral and maxillofacial surgery, 2nd ed. Volume 1. London, BC Decker, 2002

13. Roy KM, Bagg J. Antibiotic prescribing by general dental practitioners in the greater Glasgow Health Board, Scotland. British Dental Journal, 2000, 188:674-676.
14. Lewis MAO, MacFarlane TW, McGowan DA. A microbiological and clinical review of the acute dento-alveolar abscess. British Journal of Oral \& Maxillofacial Surgery, 1990, 28:359-366.

15. Lewis MA et al. Prevalence of penicillin resistant bacteria in acute suppurative oral infection. Journal of Antimicrobial Chemotherapy, 1995, 35:785-791.

16. Thomas DW et al. Antibiotic prescription for acute dental conditions in the primary care setting. British Dental Journal, 1996, 181:401-404

17. Palmer NAO, Martin MV. An investigation of antibiotic prescribing by general dental practitioners: a pilot study. Primary Dental Care, 1998, 5:11-14.

18. Slots J. Systemic antibiotics in periodontics. Journal of Periodontology, 1996, 67:831-838.

19. Martin MV et al. Acute dentoalveolar infections: an investigation of the duration of antibiotic therapy. British Dental Journal, 1997, 183:135-137.

20. Sots J, Pallasch TJ. Dentists' role in halting antimicrobial resistance. Journal of Dental Research, 1996, 75:1338-1341.

21. Askarian M, Reza Moravveji A, Assadian O. Prescription of prophylactic antibiotics for neurosurgical procedures in teaching hospitals in Iran. American Journal of Infection Control, 2007, 35:260-262.

22. Askarian $\mathrm{M}$ et al. Adherence to American Society of HealthSystem Pharmacists surgical antibiotic prophylaxis guidelines in Iran. Infection Control and Hospital Epidemiology, 2006, 27:876-878

23. Askarian M, Moravveji AR, Etminan M. Prophylactic antibiotic therapy in gynecologic-obstetric procedures: experience from three Iranian teaching hospitals. Indian Journal of Medical Sciences, 2006, 60:245-246.

24. Steed $M$, Gibson J. An audit of antibiotic prescribing in general dental practice. Primary Dental Care, 1997, 4:66-70.

25. Palmer NA, Daily YM, Martin MV. Can audit improve antibiotic prescribing in general dental practice? British Dental Journal, 2001, 191:293-295. 